\title{
TRATAMENTO ELETROQUÍMICO DE EFLUENTE DA PRODUÇÃO DE BIODIESEL USANDO UM ELETRODO DO TIPO ADE: $\mathrm{Ti} / \mathrm{IrO}_{2}-\mathrm{Nb}_{2} \mathrm{O}_{5}$
}

Carlos H. M. Fernandes ${ }^{a}$, Marcelo M. Yamasaki ${ }^{a}$, Fernando L. Silva ${ }^{b}$, Vanessa M. Vasconcelos ${ }^{b}$, Robson S. Rocha ${ }^{b}$, Marcos R. V. Lanza ${ }^{\mathrm{b}, *}$, Marilza Castilho ${ }^{\mathrm{a}}$, Evandro L. Dall'Óglio ${ }^{\mathrm{a}}$ e Ailton J. Terezo ${ }^{\mathrm{a}, *}$

a'Departamento de Química, Universidade Federal do Mato Grosso, Av. Fernando Corrêa da Costa, 2367, Boa Esperança, 78090600 Cuiabá - MT, Brasil

'Instituto de Química de São Carlos, Universidade de São Paulo, 13560-970, São Carlos - SP, Brasil

Recebido em 22/04/2017; aceito em 26/09/2017; publicado na web em 04/12/2017

\begin{abstract}
ELECTROCHEMICAL TREATMENT OF WASTEWATER FROM BIODIESEL PRODUCTION USING A DSA KIND ELECTRODE: $\mathrm{Ti} / \mathrm{IrO}_{2}-\mathrm{Nb}_{2} \mathrm{O}_{5}$. This work presents the electrochemical treatment of wastewater of biodiesel production, using DSA based on $\mathrm{IrO}_{2}$ and $\mathrm{Nb}_{2} \mathrm{O}_{5}$. The anode was prepared by modified Pechini method and characterized by scanning electron microscopy (SEM) and X-ray diffraction (XRD), in order to evaluate structural and morphological properties and by electrochemical impedance spectroscopy, cyclic voltammetry and accelerated stability test to investigate the electrochemical behavior. The SEM image shows the typical mud-cracked layer. By the XRD measurements, the oxides onto the support were crystallized as tetragonal $\mathrm{IrO}_{2}$ and hexagonal $\mathrm{Nb}_{2} \mathrm{O}_{5}$. The amount of glycerin and methanol in the wastewater were 1.60 and $0.31 \%(\mathrm{w} / \mathrm{w})$, respectively. The electrochemical treatment of the wastewater was evaluated by electrolysis at different current densities and time. The total organic carbon (TOC) and UV-Vis spectra were performed during the electrolysis. The UV-Vis spectra obtained during the electrolysis at 100 and $150 \mathrm{~mA} \mathrm{~cm}^{-2}$, shows the disappearance of an absorption band at $264 \mathrm{~nm}$ and the appearance of one at $290 \mathrm{~nm}$, which the absorption increases as current and the time of the electrolysis increases. The electrochemical treatment with the proposed DSA leads to $80 \%$ of removal of TOC by electrolysis at $150 \mathrm{~mA} \mathrm{~cm}^{-2}$ during the first 6 hours of the electrochemical treatment.
\end{abstract}

Keywords: biodiesel; wastewater; electrochemical treatment; DSA.

\section{INTRODUÇÃO}

O aumento da demanda por energia associado à limitação de fontes energéticas têm levado ao desenvolvido de estratégias para o uso de fontes alternativas de energia, como os biocombustíveis. Estudos têm mostrado que o uso de biocombustíveis reduz a emissão de gases tóxicos relacionados ao efeito estufa, como $\mathrm{HCs}, \mathrm{CO}$ e $\mathrm{CO}_{2}$, particulados de matéria orgânica e óxidos sulfurosos responsáveis pela chuva ácida. ${ }^{1} \mathrm{O}$ biodiesel é produzido a partir de óleos vegetais, gordura animal, óleos e gorduras residuais que, na presença de um catalisador ácido, básico ou enzimático, reage com álcool de cadeia curta, metanol ou etanol, resultando em ésteres de ácidos graxos de cadeia longa. ${ }^{2}$ Na produção industrial do biodiesel é necessário um excesso de álcool para promover maior rendimento na reação. ${ }^{3}$ O biodiesel é formado em um mecanismo reversível de três etapas consecutivas, onde o triglicerídeo é convertido a diglicerídeos, monoglicerídeos e glicerol, principal coproduto da obtenção do biodiesel, resultando em 3 mols de ésteres para cada 1 mol de triglicerídeo. ${ }^{4}$

Nos últimos anos, o biodiesel tornou-se uma fonte de energia alternativa promissora mundialmente, sendo que os E.U.A. ocupam a $1^{\text {a }}$ posição no ranking mundial, com produção de $5.927 .952 \mathrm{~m}^{3}$ no ano 2016. O Brasil ocupa a $2^{\mathrm{a}}$ posição neste ranking e, segundo dados da Agência Nacional de Petróleo, Gás Natural e Biocombustível (ANP), foram produzidos $3.801 .339 \mathrm{~m}^{3}$ de biodiesel no ano de 2016 , sendo $1.646 .828 \mathrm{~m}^{3}$ de biodiesel somente na região do Centro-Oeste, a maior produtora do Brasil, com 43,3\% do total produzido. ${ }^{5}$ Atualmente, a legislação brasileira determina a mistura compulsória de $8 \%$ em volume de biodiesel ao óleo diesel rodoviário, formando o que se denomina a mistura B8, e alcançará a mistura B10 em março de 2019, conforme estabelecido pela Lei No 13.263 , de 23 de março de 2016.

*e-mail: ajterezo@ufmt.br; marcoslanza@iqsc.usp.br
Embora o biodiesel seja considerado uma fonte renovável, estudos apontam que para cada $\mathrm{m}^{3}$ de biodiesel produzido são necessários de 0,2 a 1,2 $\mathrm{m}^{3}$ de água na etapa de lavagem e purificação, o que resulta na produção de grande quantidade de efluente. ${ }^{6-8}$ Neste efluente, geralmente, são encontrados óleos/gorduras, sais, catalisador remanescente, sabão, monoglicerídeos, triglicerídeos, biodiesel e álcool, em concentrações variadas. A presença destes resíduos dificulta o lançamento da água de lavagem diretamente nos corpos hídricos receptores.

Os constituintes existentes no efluente resultam em uma carga orgânica elevada quando comparado com outros tipos de efluentes. Esta elevada carga orgânica dificulta o tratamento biológico devido à falta de nutrientes como fonte de nitrogênio, necessários para metabolização, pois apenas o carbono existe como fonte de nutriente. ${ }^{2}$ No entanto, com interesse de minimizar o impacto ambiental, em algumas indústrias, ao final do processo de produção do biodiesel, o efluente é tratado e a água recuperada é reutilizada no processo produção deste biocombustível. ${ }^{1,2,6}$ Porém, mesmo com a reutilização, o volume de efluentes gerados é grande.

Atualmente, os efluentes das usinas de biodiesel têm sido tratados empregando-se processos biológicos ${ }^{7,9}$ e físico-químicos ${ }^{10,11}$ que, embora possibilitem reduções da carga orgânica poluente, não são capazes de remover contaminantes químicos, como metanol e glicerina. O metanol e a glicerina, em função de suas características químicas, são miscíveis em água e, portanto, de difícil remoção em processos de tratamentos secundários clássicos. Neste contexto, processos terciários de tratamento têm sido propostos ${ }^{12,13}$ para aumentar a remoção dos poluentes orgânicos, especificamente a glicerina e o metanol remanescentes dos processos de tratamentos secundários. Dentre os processos terciários, destaca-se os processos de tratamento eletroquímico. ${ }^{8,14,15}$

No tratamento eletroquímico de efluentes, geralmente, são utilizados diferentes materiais para a confecção dos anodos, tais 
como Ti/Pt-Ir, fibras de carbono, $\mathrm{MnO}_{2}$, feltro de carbono poroso, e carbono vítreo reticulado. ${ }^{16}$ No entanto, um dos grandes desafios é a confecção de um eletrodo estável e que apresente elevada eficiência de degradação em amostras complexas como os efluentes industriais.

Neste trabalho é apresentado um estudo do tratamento do efluente da produção de biodiesel utilizando oxidação eletroquímica com um anodo dimensionalmente estável (ADE) à base de óxidos de irídio $\left(\mathrm{IrO}_{2}\right)$ e nióbio $\left(\mathrm{Nb}_{2} \mathrm{O}_{5}\right)$.

\section{EXPERIMENTAL}

\section{Preparo e caracterização dos eletrodos ADEs}

Os eletrodos foram preparados pelo método de Pechini modificado, conforme procedimento detalhadamente descrito na literatura. ${ }^{17,18}$ Resumidamente, as resinas contendo ácido cítrico, etilenoglicol e sais precursores foram pinceladas sobre uma chapa de titânio metálica (TiBrasil 99,7\%). Os sais precursores foram $\mathrm{IrCl}_{4} \cdot \mathrm{xH}_{2} \mathrm{O}$ (Johnson Matthey) e $\mathrm{NH}_{4} \mathrm{NbO}\left(\mathrm{C}_{2} \mathrm{O}_{4}\right)_{2}$ (Sigma-Aldrich). No fluxograma mostrado no Material Suplementar (Figura 1S) encontram-se todas as etapas e condições de preparo do eletrodo.

A caracterização estrutural e morfológica dos ADEs foi realizada por microscopia eletrônica de varredura (MEV) com equipamento da Shimadzu modelo SSX-550 com radiação incidente de $25 \mathrm{kV}$ em diferentes magnificações (800x e 8000x). A estrutura cristalina foi analisada por medidas de difração de raios-X (DRX) usando um equipamento da Bruker modelo Advance D8 com radiação de $\mathrm{CuK} \alpha(\lambda=1,5418 \AA)$. O teste de estabilidade acelerado (TEA) foi realizado em solução de $\mathrm{H}_{2} \mathrm{SO}_{4} 1,0 \mathrm{~mol} \mathrm{~L}^{-1}$ a $50^{\circ} \mathrm{C}$ com aplicação de $400 \mathrm{~mA} \mathrm{~cm}^{-2}$, sendo que a estabilidade do eletrodo foi considerada o tempo até o potencial atingir $6,0 \mathrm{~V}(\mathrm{ecs})$. Com interesse em avaliar a interação entre a superfície do eletrodo e a solução, medidas de espectroscopia de impedância eletroquímica (EIE), em solução de $\mathrm{H}_{2} \mathrm{SO}_{4}$ $1,0 \mathrm{~mol} \mathrm{~L}^{-1}$, na faixa de frequência de $10^{-2}$ a $10^{5} \mathrm{~Hz}$, em potencias $\left(E_{d c}\right)$ na região de carregamento $(0,6 \mathrm{~V})$ e na região de transferência de carga da $\operatorname{RDO}(1,2,1,3$ e 1,4V), foram realizadas usando um analisador de resposta em frequência da Autolab mod. FRA2 com perturbação pico-a-pico de 5,0 $\mathrm{mV}$. Os voltamogramas cíclicos foram obtidos antes e após o TEA em solução de $\mathrm{H}_{2} \mathrm{SO}_{4} 1,0 \mathrm{~mol} \mathrm{~L}{ }^{-1}$ em uma faixa de potencial de 0,15 a $1,15 \mathrm{~V}$ (ecs) com velocidade de varredura de $20 \mathrm{mV} \mathrm{s}^{-1}$ em uma célula de compartimento único. Utilizou-se como eletrodo auxiliar uma placa de platina com $6,25 \mathrm{~cm}^{2}$ de área e o eletrodo de referência de calomelano saturado $\left(\mathrm{Hg} / \mathrm{Hg}_{2} \mathrm{Cl}_{2} / \mathrm{KCl}_{\text {sat }}\right)$. Os experimentos de TEA e EIE foram realizados em um potenciostato/ galvanostato Autolab mod. PGSTAT30.

Para a caracterização por DRX, MEV, EIE e para o TEA foram confeccionados eletrodos de $1,0 \mathrm{~cm}^{2}$ com filme eletrocatalítico nas duas faces. Para a degradação eletroquímica dos efluentes, foi confeccionado um ADE de $4,0 \mathrm{~cm}^{2}$ depositando o filme eletrocatalítico em uma face.

\section{Tratamento eletroquímico do efluente}

A amostra de efluente da produção de biodiesel foi cedida por uma usina situada no estado de Mato Grosso, cujos parâmetros físico-químicos ${ }^{19}$ estão apresentados na Tabela $1 \mathrm{~S}$ do Material Suplementar. Todos os reagentes utilizados eram de grau analítico, sendo utilizados sem nenhuma purificação prévia. O eletrólito suporte utilizado foi $\mathrm{K}_{2} \mathrm{SO}_{4} 0,05 \mathrm{~mol} \mathrm{~L}^{-1}$. Todas as soluções foram preparadas com água ultrapura $(18,2 \mathrm{M} \Omega \mathrm{cm})$ que foi processada por um sistema de purificação Milli-Q Gradient da Millipore.

Os experimentos de eletrólise do efluente de biodiesel foram realizados em um reator de polipropileno aberto contendo $180 \mathrm{~mL}$ de solução sob agitação em $500 \mathrm{rpm}$, temperatura de $25^{\circ} \mathrm{C}$, contendo o ADE a base de óxidos de $\mathrm{IrO}_{2}$ e $\mathrm{Nb}_{2} \mathrm{O}_{5}$ com área total de $4,0 \mathrm{~cm}^{2}$. Um equipamento potenciostato/galvanostato PGSTAT-302N da Autolab foi usado para medidas de eletrólises. As eletrólises foram acompanhadas por medidas espectrométricas na faixa de 200-800 nm em um equipamento Varian modelo Cary 50. As concentrações de matéria orgânica do efluente foram determinadas pela oxidação desta matéria em um equipamento Shimadzu TOC-VCPN. Durante o processo eletroquímico, houve a avaliação de diferentes densidades de corrente (j): 25, 50, 100 e $150 \mathrm{~mA} \mathrm{~cm}^{-2}$ durante $4 \mathrm{~h}$, e uma com aplicação de $150 \mathrm{~mA} \mathrm{~cm}^{-2}$ durante $8 \mathrm{~h}$.

\section{RESULTADOS E DISCUSSÃO}

\section{Caracterização estrutural do $\mathrm{ADE}$ a base de $\mathrm{IrO}_{2} \mathrm{e} \mathrm{Nb}_{2} \mathrm{O}_{5}$}

A caracterização morfológica e estrutural do eletrodo foi realizada por medidas de DRX e MEV. Na Figura 1 apresenta-se o padrão de DRX do ADE de óxidos de $\mathrm{IrO}_{2}$ e $\mathrm{Nb}_{2} \mathrm{O}_{5}$ e podem ser observadas estruturas cristalinas características para este tipo de eletrodo. ${ }^{20,21}$ O padrão de DRX está bem definido para picos de titânio metálico, óxido de irídio e irídio metálico. Picos de titânio metálico em $40,1^{\circ}$ e $53^{\circ}$ foram encontrados e indexados com padrão ICSD N ${ }^{\circ} 181718$, picos de $\mathrm{IrO}_{2}$ em $27,7^{\circ}$ e $34,6^{\circ}$ na forma tetragonal também foram encontradas e indexadas com a ficha do ICSD $N^{\circ} 84577$ e picos de irídio metálico em $40,7^{\circ}$ e $47,2^{\circ}$ foram encontrados e indexados com a ficha cristalográfica do ICSD N $\mathrm{N}^{\mathrm{0}} 41524$.

Não foram observados picos relacionados à estrutura cristalina de óxido de nióbio, $\mathrm{Nb}_{2} \mathrm{O}_{5}$, que pode estar relacionado ao fato de que a temperatura empregada durante o processo de calcinação pode não ter sido suficiente para formar sua fase cristalina, deixando sua estrutura amorfa, conforme evidenciado pelo alargamento dos picos de difração na região de $27^{\circ}$ e $35^{\circ}$. O comportamento obtido reflete o processo de transporte de massa, responsável pelo aumento da cristalinidade. Embora a cristalinidade resulte em diminuição da capacidade de carregamento da dupla-camada elétrica, consequentemente menor número de sítios eletroquimicamente ativos, um efeito importante correlacionado é o aumento da estabilidade do eletrodo durante a eletrólise. ${ }^{22}$

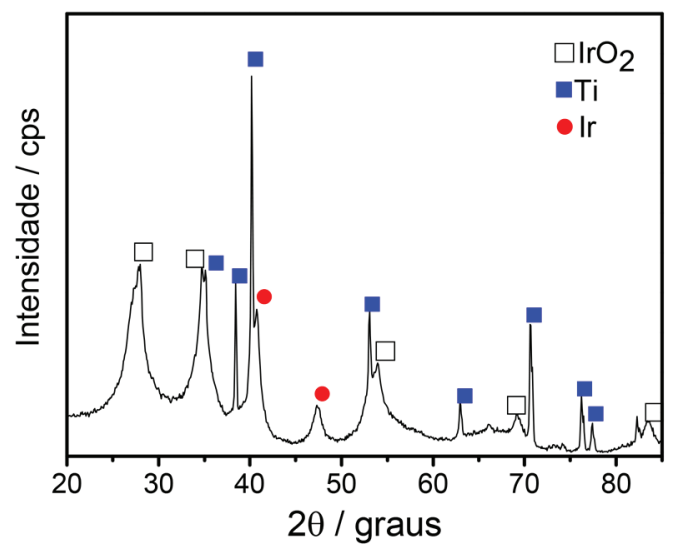

Figura 1. Difratograma de raio-x do ADE de $\mathrm{Ti} / \mathrm{IrO}_{2}-\mathrm{Nb}_{2} \mathrm{O}_{5}(60: 40 \mathrm{~mol} \%)$ calcinado a $500{ }^{\circ} \mathrm{C}$

Na Figura 2 estão apresentadas as imagens de MEV do ADE, nas quais se observa uma morfologia característica chamada de "barro rachado" formada em função do processo de tratamento térmico drástico que as amostras são submetidas, que promove a rápida saída de gases $\mathrm{CO}_{2}$ original da decomposição da matéria orgânica. .7,20-22 $^{2}$ 


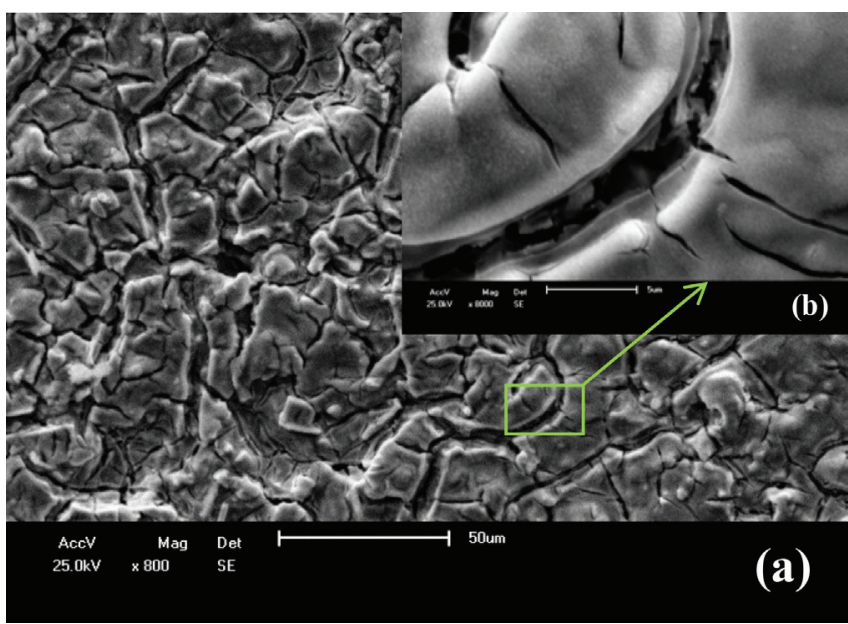

Figura 2. Imagens de $\mathrm{MEV}$ do $\mathrm{ADE}$ a base de $\mathrm{IrO}_{2}$ e $\mathrm{Nb}_{2} \mathrm{O}_{5}$ com magnificação de (a) 800x e (b) 8000x no detalhe

\section{Caracterização eletroquímica}

A Figura 3 mostra os espectros de impedância eletroquímica para o eletrodo aplicando-se diferentes potencias durante as medidas. Observa-se que o eletrodo recém-preparado apresenta comportamento capacitivo no potencial da dupla camada elétrica $(0,6 \mathrm{~V})$ e inclinação próxima a $90^{\circ}$. Entretanto, ao aplicar o potencial de $1,2 \mathrm{~V}$, o diagrama no plano complexo mostra uma curvatura em regiões de baixa frequência, indicando o início da formação do semicírculo. ${ }^{17}$ Este comportamento indica o início da ocorrência da reação de desprendimento de oxigênio (RDO). A resposta de impedância foi aproximada ao modelo de circuitos equivalentes mostrado na inserção da Figura 3, que reflete um eletrodo não polarizável e permite a estimativa dos valores de capacitância da dupla-camada elétrica e da resistência de transferência de carga. ${ }^{17}$ Em detalhe na Figura 3, a ampliação na região de alta frequência do digrama de impedância mostra a formação do semicírculo nos potenciais de 1,3 e 1,4 V. Para estes potenciais a resistência de transferência de carga para a RDO está, respectivamente, em torno de 3,2 e 1,2 k $\Omega$. Desta forma, os espectros de impedância na região com $\mathrm{E} \geq 1,2 \mathrm{~V}$ mostram que os ADEs a base de óxidos de $\mathrm{IrO}_{2}$ e $\mathrm{Nb}_{2} \mathrm{O}_{5}$ comportam-se como ativos para RDO. ${ }^{17}$

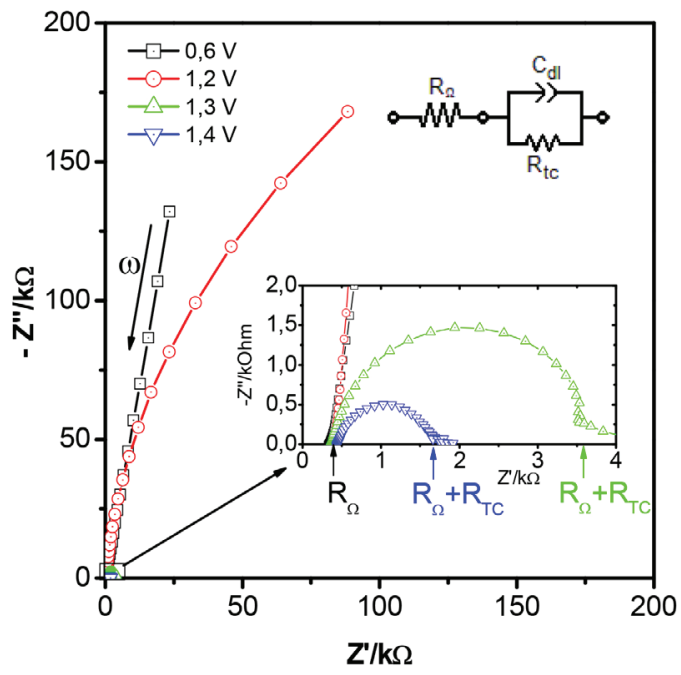

Figura 3. Espectros de impedância no plano complexo do ADE em diferentes potenciais em $\mathrm{H}_{2} \mathrm{SO}_{4} 1,0 \mathrm{~mol} \mathrm{~L}^{-1}$. Inserção: Modelo de circuito equivalente utilizado para obtenção dos valores aproximados de resistência de transferência de carga
Com interesse em avaliar a durabilidade do ADE foi realizado teste de estabilidade acelerado, ${ }^{23}$ conforme é mostrado na curva de potencial (E) versus tempo (t) da Figura 4. Pode-se observar que o potencial permanece constante com o progresso da eletrólise até o período de $160 \mathrm{~h}$, onde o potencial é $2,0 \mathrm{~V}$ (ecs), e atinge 2,7 V(ecs) em $170 \mathrm{~h}$ de TEA, mostrando que o eletrodo é bastante resistente em função do tempo de eletrólise. Ao final de $171 \mathrm{~h}$ o potencial do eletrodo aumenta bruscamente até $6,0 \mathrm{~V}(\mathrm{ecs})$ e o eletrodo é considerado inativo, devido ao aumento da queda ôhmica e desgaste contínuo após um longo período de eletrólise. É importante ressaltar que o TEA é uma medida em escala de laboratório que visa avaliar a durabilidade do eletrodo. Destaca-se que o valor de $171 \mathrm{~h}$ para o ADE preparado neste trabalho é maior que muitos na literatura. ${ }^{20,21}$ Inserido na Figura 4 estão os voltamogramas cíclicos do ADE antes e depois do TEA. Analisando-se os VCs verifica-se comportamento pseudo-capacitivo relativo ao processo de dupla-injeção de cargas associado à transição redox de estado sólido do $\operatorname{Ir}(\mathrm{III}) / \mathrm{Ir}(\mathrm{IV}) \cdot{ }^{17,20-22}$ Após o TEA verifica-se diminuição da densidade de corrente no eletrodo, devido à redução de sítios eletroativos no ADE. Além disso, verifica-se a mudança do comportamento voltamétrico típico de um pseudo-capacitor para um eletrodo passivante, evidenciando a inativação do ADE pela dissolução eletrolítica do óxido metálico $\left(\mathrm{IrO}_{2}\right) \cdot{ }^{20-22}$

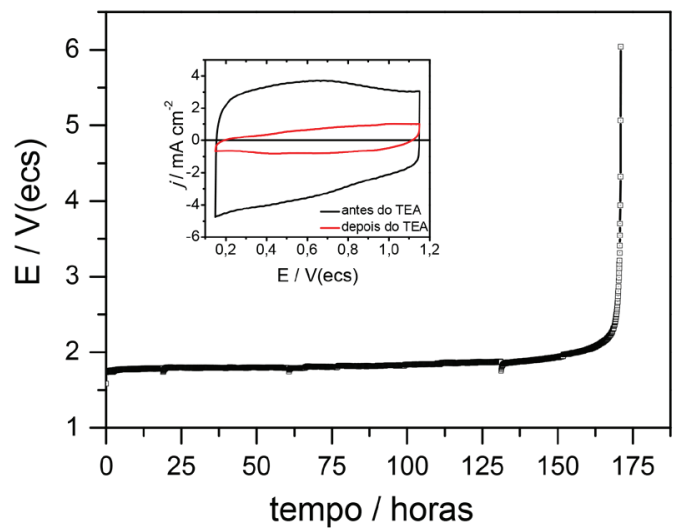

Figura 4. Curva de potencial ( $E)$ versus tempo ( $t$ ) para o TEA do ADE a base de óxidos de $\mathrm{IrO}_{2}$ e $\mathrm{Nb}_{2} \mathrm{O}_{5} \mathrm{em} \mathrm{H}_{2} \mathrm{SO}_{4} 1,0 \mathrm{~mol} \mathrm{~L}{ }^{-1}, 50{ }^{\circ} \mathrm{C} \mathrm{e} \mathrm{j=} 400 \mathrm{~mA} \mathrm{~cm}^{-2}$. Inserção: Voltamogramas cíclicos do ADE antes e após o TEA

Em função dos resultados da caracterização morfológica, estrutural e eletroquímica dos ADE, o efluente da usina de biodiesel foi submetido ao tratamento eletroquímico, já que os resultados mostraram que o ADE é considerado ativo e um bom candidato para reação de desprendimento de oxigênio, o que pode possibilitar o tratamento eletroquímico dos efluentes.

\section{Tratamento eletroquímico do efluente de biodiesel}

O tratamento eletroquímico do efluente da produção de biodiesel foi estudado por eletrólise usando ADE a base de óxidos de $\mathrm{IrO}_{2}$ e $\mathrm{Nb}_{2} \mathrm{O}_{5}$. Antes e após o tratamento eletroquímico, o efluente foi caracterizado por medidas de carbono orgânico total (COT) e espectrofotometria UV-Vis. Os parâmetros físico-químicos do efluente coletado na usina estão apresentados na Tabela $1 \mathrm{~S}$ em informação complementares. Destes parâmetros, destaca-se os teores de glicerina e metanol no efluente, respectivamente, $1,60 \%$ e $0,31 \%(\mathrm{~m} / \mathrm{m})$.

A Figura 5 mostra a porcentagem de redução de COT em função da densidade de corrente aplicada na faixa de 25 a $150 \mathrm{~mA} \mathrm{~cm} \mathrm{cu}^{-2}$ rante $4 \mathrm{~h}$ de eletrólise. Pode-se observar que o aumento da densidade de corrente provocou um aumento na redução da carga orgânica em função do tempo. 


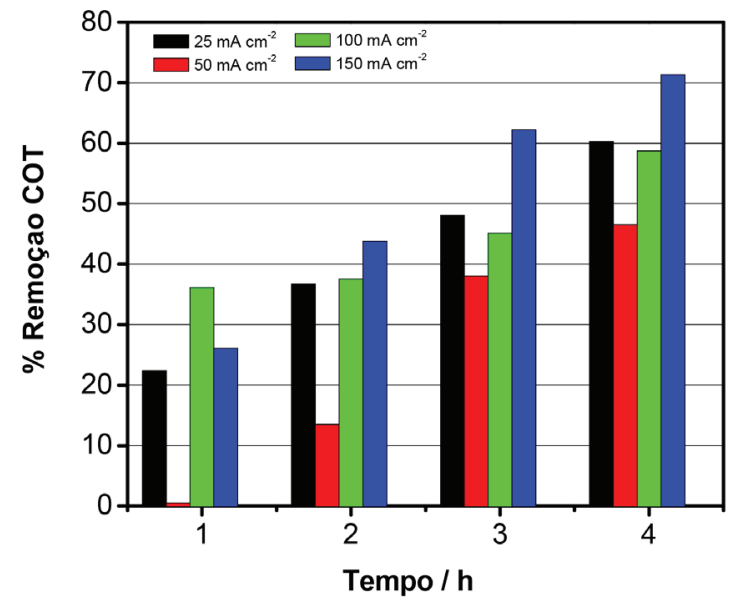

Figura 5. Efeito da densidade de corrente $j$ na \% de redução de COT em função do tempo de eletrólise do efluente de biodiesel $+\mathrm{K}_{2} \mathrm{SO}_{4} 0,05 \mathrm{~mol} \mathrm{~L}^{-1}$

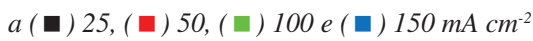

Aplicando-se densidades de corrente de 25, 50 e $100 \mathrm{~mA} \mathrm{~cm}{ }^{-2}$, durante 4 horas de eletrólise, a porcentagem de matéria orgânica removida foi, respectivamente, 53, 48 e $60 \%$. Um aumento da redução de COT para 71,5\% foi observado com densidades de correntes de $150 \mathrm{~mA} \mathrm{~cm}^{-2}$. Com eletrólises realizadas em um tempo de até $8 \mathrm{~h}$ aplicando-se densidades de corrente de $150 \mathrm{~mA} \mathrm{~cm}^{-2}, 79 \%$ de redução foi alcançado em 6 h de eletrólise, permanecendo com $80 \%$ de redução de COT até 8 h de eletrólise.

De acordo com estabelecido na literatura, a oxidação da matéria orgânica presente em efluentes está relacionada, principalmente, à oxidação direta devido ao par redox $\mathrm{MO} / \mathrm{MO}_{\mathrm{x}+1}$, característico de eletrodos compostos de óxidos de irídio com altos estados de oxidação. ${ }^{24-26}$ A oxidação direta é favorecida quando a espécie contaminante se encontrar em elevadas concentrações, o que ocorre de forma inversa com a oxidação indireta ou mediada por radical hidroxila adsorvido na superfície do eletrodo, reagindo com os compostos orgânicos.

A eficiência de corrente $\left(\mathrm{E}_{f} \mathrm{C}\right)$ neste trabalho foi avaliada a partir das medidas de COT e foi calculada usando a equação (1): ${ }^{25}$

$$
\mathbf{E}_{\mathbf{f}} \mathbf{C}=\frac{2,67\left[(\mathbf{C O T})_{\mathbf{t}}-(\mathbf{C O T})_{\mathbf{t}+\Delta t}\right] \mathbf{F V}}{8 \mathbf{I} \Delta \mathbf{t}}
$$

em que o valor 2,67 corresponde à razão entre a massa molar do oxigênio molecular e do átomo de carbono, a variação do $\mathrm{COT}_{0}$ e o $\mathrm{COT}_{\mathrm{t}+\Delta \mathrm{t}}$ são o COT $\left(\mathrm{g} \mathrm{L}^{-1}\right)$ nos tempos 0 e t $(\mathrm{h})$, respectivamente. F é a constante de Faraday, V é o volume de solução (L), I é a corrente aplicada (A) e $t$ o tempo (h).

O consumo energético (CE) foi calculado pela equação (2):

$$
C E=\frac{U I t}{V}
$$

em que $I$ é a corrente aplicada (A), U é o potencial de operação (V), t é o tempo de eletrólise (h) e $\mathrm{V}$ é o volume da solução.

$\mathrm{Na}$ Tabela 1 são apresentados os valores de $\mathrm{E}_{f} \mathrm{C}, \mathrm{CE}$ e \% de redução de COT em diferentes densidades de corrente. Pode ser observado que o $\mathrm{CE}$ é inversamente proporcional à $\mathrm{E}_{f} \mathrm{C}$. Este comportamento pode estar relacionado ao processo de transferência de massa e competição entre a reação de desprendimento de oxigênio e a eletrooxidação da matéria orgânica. ${ }^{24}$ Avaliando-se a $\mathrm{E}_{f} \mathrm{C}$ em função da densidade de corrente, observa-se uma variação da eficiência de corrente de 1,74 a 0,30 aplicando densidades de corrente que variaram de $25 \mathrm{~mA} \mathrm{~cm}$ a $^{-2} 150 \mathrm{~mA} \mathrm{~cm}^{-2}$ com 4 horas e 0,13 com aplicação de $150 \mathrm{~mA} \mathrm{~cm}^{-2}$ ao final de $8 \mathrm{~h}$ de eletrólise. Estes resultados mostram que para atingir um maior decaimento da concentração dos compostos orgânicos, com baixo consumo energético, o sistema eletroquímico pode ser operado em baixas densidades de corrente, entretanto este processo leva a um período maior de tratamento.

Tabela 1. Resultados da EC, CE e da \% de redução de COT após 4h de eletrólise

\begin{tabular}{cccc}
\hline $\mathrm{j} / \mathrm{mA} \mathrm{cm}^{-2}$ & $\mathrm{E}_{f} \mathrm{C}$ & $\mathrm{CE} / \mathrm{kWh} \mathrm{m}^{-3}$ & $\begin{array}{c}\text { Redução de } \\
\text { COT }(\%)\end{array}$ \\
\hline 25 & 1,70 & 11,98 & 60,3 \\
50 & 0,52 & 35,50 & 46,5 \\
100 & 0,45 & 114,4 & 58,7 \\
150 & 0,30 & 221,3 & 71,3 \\
$150^{\#}$ & 0,13 & 412,2 & 79,0 \\
\hline
\end{tabular}

\#eletrólise exaustiva a $150 \mathrm{~mA} \mathrm{~cm}^{-2}$ por 8 horas.

Na Figura 6 é mostrada a $\mathrm{E}_{f} \mathrm{C}$ e CE em função da $j$, após 4 h de eletrólise. Observa-se que o aumento do CE diminui exponencialmente a $\mathrm{E}_{f} \mathrm{C}$. Com aplicação de $25 \mathrm{~mA} \mathrm{~cm}^{-2}$ o consumo energético chegou aproximadamente a $36 \mathrm{kWh} \mathrm{m}^{-3}$. Entretanto, a partir de 50 até $150 \mathrm{~mA} \mathrm{~cm}^{-2}$ observa-se um aumento significativo do consumo energético de 36 para $221 \mathrm{kWh} \mathrm{m}^{-3}$. No entanto, esse valor máximo de CE é ainda menor que outros relatados recentemente para a oxidação eletroquímica de poluentes orgânicos..$^{25-28}$ Os experimentos realizados com aplicação de $25 \mathrm{~mA} \mathrm{~cm}^{-2}$ tem menor consumo energético, demonstrando que estes experimentos podem ter uma melhor eficiência em baixas densidades de correntes.

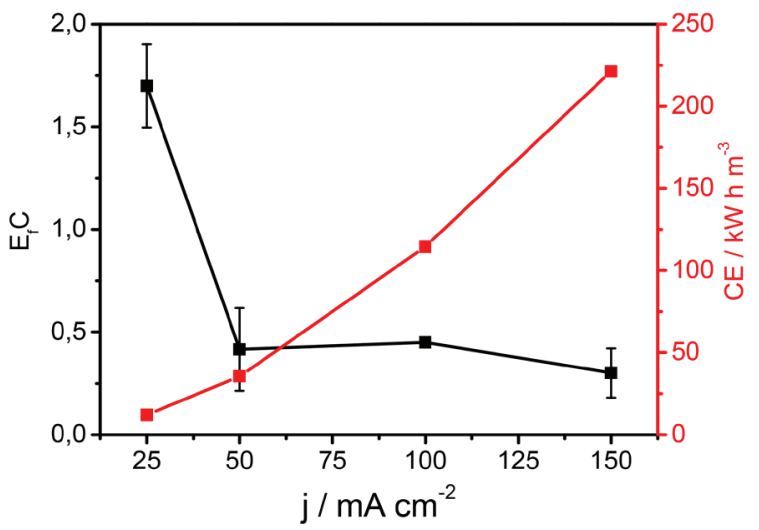

Figura 6. Relação de efeitos entre a densidade de corrente (j), eficiência de corrente ( ) e consumo energético ( $\mathbf{\square}$ ) após 4 horas de eletrólise do efluente de Biodiesel $+\mathrm{K}_{2} \mathrm{SO}_{4} 0,05 \mathrm{~mol} \mathrm{~L} \mathrm{~L}^{-1}$ e $\mathrm{T}=25^{\circ} \mathrm{C}$

Durante os experimentos de degradação eletroquímica, alíquotas do efluente foram coletadas e monitoradas, realizando medidas de espectros na região do ultravioleta-visível como mostrados na Figura 7 (todas as amostras foram diluídas 10x em água deionizada).

O espectro na Figura 7a mostra inicialmente que há um pico de absorção por volta de $260 \mathrm{~nm}$. Durante a aplicação de $25 \mathrm{~mA} \mathrm{~cm}{ }^{-2}$ não foi observado o surgimento de novos picos na região estudada, devido desdobramentos relacionados à formação de outros produtos secundários em detrimento aos compostos orgânicos existentes nos efluentes. No entanto, ocorre uma diminuição da absorbância ao longo de $240 \mathrm{~min}$, iniciando em 0,346 e terminando em torno de 0,180 , aproximadamente, em destaque na Figura 7(a). 

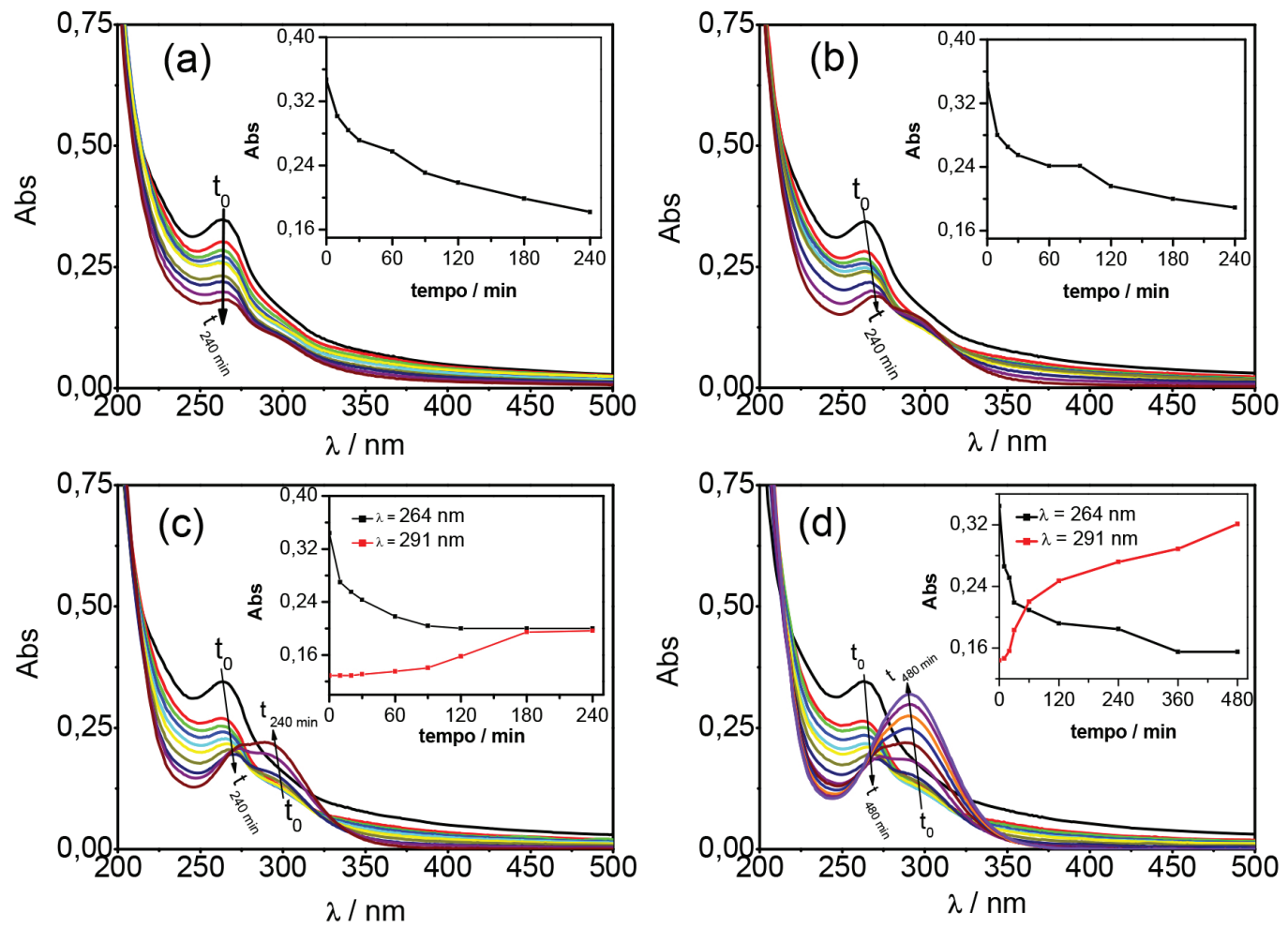

Figura 7. Espectros UV-Vis durante a eletrólise do efluente de Biodiesel em diferentes densidades de corrente (a) 25 , (b) 50 , (c) 100 mA cm-2 durante 4 h de eletrólise e (d) $150 \mathrm{~mA} \mathrm{~cm}^{-2}$ durante 8 h de eletrólise

Para os espectros com aplicação de densidade de corrente de $50 \mathrm{~mA} \mathrm{~cm}$ c $^{-2}$ é possível observar que houve uma pequena mudança no espectro ultravioleta visível, Figura 7(b), com surgimento de bandas de absorção em torno de $290 \mathrm{~nm}$ com absorbância em torno de 0,136 . Observa-se, ainda, que nos primeiros 10 min de degradação houve um maior aumento no decaimento da absorbância, em relação ao experimento com aplicação de $25 \mathrm{~mA} \mathrm{~cm}^{-2}$. Observa-se, também, o surgimento de uma banda em torno de $290 \mathrm{~nm}$, porém, sem muita intensidade e definição.

Os espectros obtidos na eletrólise com $100 \mathrm{~mA} \mathrm{~cm}^{-2}$ estão apresentados na Figura 7c. Estes espectros mostram o decaimento da banda em 264 nm e o surgimento de uma nova banda de absorção em aproximadamente $290 \mathrm{~nm}$. Observa-se também que o decaimento da banda característica do efluente em $260 \mathrm{~nm}$ foi mais acentuado na primeira hora de eletrólise, tornando-se quase constante em relação aos demais tempos. Nos espectros obtidos durante a eletrólise a 150 $\mathrm{mA} \mathrm{cm} \mathrm{c}^{-2}$ por $8 \mathrm{~h}$ (Figura $7 \mathrm{~d}$ ) verificou-se o mesmo comportamento, observado para densidade de $100 \mathrm{~mA} \mathrm{~cm}^{-2}$, com decaimento da banda em $264 \mathrm{~nm}$ e o surgimento banda em $290 \mathrm{~nm}$ e aumento de intensidade desta banda com o tempo de eletrólise.

Portanto, este comportamento indica que as espécies químicas responsáveis pela absorção em $290 \mathrm{~nm}$ são formadas em maiores densidades de corrente e se intensificam com o tempo de eletrólise. Uma hipótese é que esta banda pode ser atribuída à transição $n \rightarrow \pi^{*}$ de ácidos carboxílicos, como ácido glicérico, fórmico, tartrônico, malônico ou ácido oxálico, espécies intermediárias ou produtos da oxidação eletroquímica da glicerina ${ }^{29}$ presentes no efluente. Além disso, outra hipótese seria a formação de espécies como acroleína e ácido acrílico que, embora são produtos de oxidação da glicerina e também resultam no deslocamento batocrômico do tipo $n \rightarrow \pi^{*}$ de ligação $\alpha, \beta$-insaturada, são instáveis nas condições da eletrólise utilizadas e, segundo dados da literatura, ocorrem em torno de $340 \mathrm{~nm} .{ }^{30}$ No entanto, as espécies químicas intermediárias ou produtos de reação responsáveis pelas mudanças nos espectros de absorção só podem ser identificadas com uso de técnicas hifenadas de cromatografia com detecção por espectroscopia de massas ou ressonância magnética nuclear.

\section{CONCLUSÃO}

O eletrodo a base de $\mathrm{IrO}_{2}$ e $\mathrm{Nb}_{2} \mathrm{O}_{5}$ apresentou elevada estabilidade nos ensaios de TEA durante 171 horas. A caracterização microestrutural mostrou a formação dos óxidos de $\mathrm{IrO}_{2} \mathrm{e} \mathrm{Nb}_{2} \mathrm{O}_{5}$. A partir dos resultados de espectroscopia de impedância eletroquímica verificou-se que o eletrodo é ativo para a reação de desprendimento de oxigênio, com baixo sobrepotencial. No estudo do tratamento eletroquímico do efluente, aplicando-se densidades de corrente de $150 \mathrm{~mA} \mathrm{~cm}{ }^{-2}$ durante 6 h, 79\% de COT foi removido. Nesta densidade de corrente, bem como a $100 \mathrm{~mA} \mathrm{~cm}^{-2}$, observou-se o surgimento de uma banda de absorção em $290 \mathrm{~nm}$, que pode estar relacionada aos intermediários da oxidação da glicerina presente no efluente. Os resultados de eficiência de corrente $\left(\mathrm{E}_{\mathrm{f}} \mathrm{C}\right)$ e consumo energético $(\mathrm{CE})$ mostram-se compatíveis com processos eletroquímicos terciários de tratamento de efluente. Estudos futuros com eletrodos de Ti/ $/ \mathrm{IrO}_{2}-\mathrm{Nb}_{2} \mathrm{O}_{5}$ em diferentes proporções de $\mathrm{Nb}_{2} \mathrm{O}_{5}$ podem resultar na melhoria destes parâmetros de eficiência energética, pois a competição com a RDO pode ser minimizada. Desta forma, além do consumo energético, a elucidação dos subprodutos formados durante a eletrólise é primordial para otimização do processo de tratamento eletroquímico visando a redução da carga poluente no efluente da produção de biodiesel pela rota da transesterificação metílica, responsável por mais de $95 \%$ da produção de biodiesel no Brasil.

\section{MATERIAL SUPLEMENTAR}

A Figura 1S relativa ao fluxograma das etapas de preparação do eletrodo, bem como a Tabela $1 \mathrm{~S}$ com os parâmetros físico-químicos do efluente de biodiesel, podem ser acessadas livremente em http:// quimicanova.sbq.org.br/ 


\section{AGRADECIMENTOS}

Os autores agradecem ao CNPq e à CAPES. A. J. Terezo agradece à FINEP/Secitec-MT Convênio 01.08.0651-00 e à FAPEMAT Proc. $569157 / 2014$ pelos suportes financeiros.

\section{REFERÊNCIAS}

1. Ma, F.; Hanna, M. A.; Bioresour. Technol. 1999, 70, 1.

2. Suarez, P. A.; Meneghetti, S. M. P.; Meneghetti, M. R.; Wolf, C. R.; Quim. Nova 2007, 30, 667.

3. Da Silva, A. C. H.; Kuhnen, C. A.; Da Silva, S. C.; Dall'oglio, E. L.; De Sousa, P. T.; Fuel 2013, 107, 387

4. Komers, K.; Skopal, F.; Stloukal, R.; Machek, J.; Eur. J. Lipid Sci. Technol. 2002, 104, 728.

5. http://www.anp.gov.br/wwwanp/dados-estatisticos, acessada em novembro de 2017

6. Van Gerpen, J.; Shanks, B.; Pruszko, R.; Clements, D.; Knothe, G.; Biodiesel Production Technology, National Renewable Energy Laboratory: Cole Boulevard, 2004.

7. Suehara, K.; Kawamoto, Y.; Fujii, E.; Kohda, J.; Nakano, Y.; Yano, T.; J. Biosci. Bioeng. 2005, 100, 437

8. Palomino Romero, J. A.; Cardoso Junior, F. S. S.; Figueiredo, R. T.; Silva, D. P.; Cavalcanti, E. B.; Sep. Sci. Technol. 2013, 48, 2073.

9. Siles, J. A.; Martín, M. A.; Chica, A. F.; Martín, A.; Bioresour. Technol. 2010, 101, 6315 .

10. Hájek, M.; Skopal, F.; Bioresour. Technol. 2010, 101, 3242.

11. Cordeiro, R. B.; Alexandre, J. I. da S.; Silva, J. P. F.; Sales, D. C. S.; Cavalcanti, L. A. P.; Revista Brasileira Gestão Ambiental e Sustentabilidade 2015, 2, 51.

12. Ngamlerdpokin, K.; Kumjadpai, S.; Chatanon, P.; Tungmanee, U.; Chuenchuanchom, S.; Jaruwat, P.; Lertsathitphongs, P.; Hunsom, M.; J. Environ. Manage. 2011, 92, 2454.
13. Feng, Y.; Yang, Q.; Wang, X.; Liu, Y.; Lee, H.; Ren, N.; Bioresour. Technol. 2011, 102, 411.

14. Pattaraluk, J.; Sangkorn, K.; Mali, H.; Energy Convers. Manage. 2010, 51,531 .

15. Srirangsan, A.; Ongwandee, M.; Chavalparit, O.; Environ. Asia 2009, 2, 15.

16. Martinez-Huitle, C. A.; Ferro, S.; Chem. Soc. Rev. 2006, 47, 1324.

17. Terezo, A. J.; Bisquert, J.; Pereira, E. C.; Garcia-Belmonte, G.; J. Electroanal. Chem. 2001, 508, 59.

18. P. Pechini. M; US Pat. 3,330,697 1967.

19. Eaton, A. D.; Franson, M. A. H.; Standard Methods for the Examination of Water \& Wastewater, 20 ${ }^{\text {th }}$ ed., American Public Health Association: Washington, DC, 1998.

20. de Oliveira-Sousa, A.; da Silva, M. A. S.; Machado, S. A. S.; Avaca, L. A.; de Lima-Neto, P.; Electrochim. Acta 2000, 47, 4467.

21. Santana, M. H. P.; De Faria, L.A.; Boodts, J. F. C.; Electrochim. Acta 2004, 49, 1935.

22. Terezo, A. J.; Pereira, E. C.; Electrochim. Acta 2000, 45, 4351.

23. Comninellis, C.; Vercesi, G. P.; J. Appl. Electrochem.1991, 21, 335-345.

24. Huitle, C. A. M.; Tese de Doutorado, Universidade de Ferrara, Itália, 2004

25. Souza, F. L.; Aquino, J. M.; Miwa, D. W.; Rodrigo, M. A.; Motheo, A. J.; J. Braz. Chem. Soc. 2014, 25, 492.

26. Polcaro, A. M.; Palmas, S.; Renoldi, F.; Mascia, M.; J. Appl. Electrochem. 1999, 29, 147.

27. Rocha, J. H. B.; Gomes, M. M. S.; Fernandes, N. S.; da Silva, D. R.; Martínez-Huitle, C.A.; Fuel Process. Technol. 2012, 96, 80.

28. Brocenschi, R. F.; Rocha-Filho, R. C.; Bochi, N.; Biaggio, S. R.; Electrochim. Acta 2016, 197, 186.

29. Fernández, P. S.; Martins, M. E.; Camara, G. A.; Electrochim. Acta 2012 , $106,180$.

30. Magneron, I.; Thévenet, R.; Mellouki, K.; Wirtz, A.; Le Bras, G.; Moortgat, G. K.; J. Phys. Chem. A 2002, 106, 2537. 